\title{
PERCEPÇÃO DE ESTUDANTES SOBRE A ATUAÇÃO DO(A) ENFERMEIRO(A) NA ATENÇÃO PRIMÁRIA À SAÚDE: REVISÃO INTEGRATIVA
}

\author{
STUDENTS' PERCEPTION OF NURSES' ROLE IN \\ PRIMARY HEALTH CARE: INTEGRATIVE REVIEW
}

\section{PERCEPCIÓN DE LOS ESTUDIANTES SOBRE EL PAPEL DE LAS ENFERMERAS EN LA ATENCIÓN PRIMARIA DE SALUD: REVISIÓN INTEGRATIVA}

\author{
Pablo Ramon Rodrigues Freitas Ramos Carloni \\ Amanda Caetano dos Santos ${ }^{2}$ \\ Flávio Adriano Borges ${ }^{3}$
}

\begin{abstract}
Como citar este artigo: Carloni PRRFR, Santos AC, Borges FA. Percepção de estudantes sobre a atuação do(a) enfermeiro(a) na Atenção Primária à Saúde: revisão integrativa. Rev baiana enferm. 2021;35:e36782.

Objetivo: analisar as evidências científicas nacionais e internacionais que abordam a percepção de estudantes de enfermagem sobre a atuação do(a) enfermeiro(a) na Atenção Primária à Saúde. Método: trata-se de revisão integrativa utilizando a base de dados da Biblioteca Virtual de Saúde e análise realizada por Estatística Textual Clássica e Classificação Hierárquica Descendente das conclusões ou considerações finais, utilizando o software IRAMUTEQ®. Resultados: foram selecionados 5 artigos dos 83 encontrados, e a análise resultou em três classes: Escassez de enfermeiros(as) para atuarem na Atenção Primária à Saúde; Formação universitária vinculada à Atenção Primária à Saúde; e Percepção dos estudantes sobre a profissão de enfermagem. Conclusão: o processo de formação e/ou produção do conhecimento em enfermagem ainda vincula a profissão a características subjetivas, apontando para a necessidade de avançarmos na direção de uma enfermagem pautada em evidências. Observou-se ainda a escassez de estudos primários abordando a temática dessa produção.
\end{abstract}

Descritores: Estudante de Enfermagem. Atenção Primária à Saúde. Estratégia Saúde da Família. Enfermagem. Revisão.

Objective: to analyze national and international scientific evidence that addresses the perception of nursing students of nurses' role in Primary Health Care. Method: this is an integrative review using the Virtual Health Library database and analysis performed by Classical Textual Statistics and Descending Hierarchical Classification from the conclusions or final thoughts, using the IRAMUTEQ ${ }^{\circledR}$ Software. Results: Of the 83 articles found, five were selected, and the analysis resulted in three classes: Scarcity of nurses to work in Primary Health Care; University education linked to Primary Health Care; and Students' perception about the nursing profession. Conclusion: the process of nursing knowledge education and/or production still links the profession to subjective characteristics, pointing to the need to advance towards evidence-based nursing. Furthermore, the research revealed scarcity of primary studies addressing the theme of this production.

Descriptors: Nursing student. Primary Health Care. Family Health Strategy. Nursing. Review.

Estudante de Enfermagem. Universidade Federal de São Carlos. São Carlos, São Paulo, Brasil. https://orcid.org/0000-0003-0347-3965.

Estudante de Enfermagem. Universidade Federal de São Carlos. São Carlos, São Paulo, Brasil. https://orcid.org/0000-0002-9795-1317.

Enfermeiro. Mestre em Gestão da Clínica. Doutor em Ciências. Professor Adjunto do Departamento de Enfermagem da Universidade Federal de São Carlos. São Carlos, São Paulo, Brasil. flavioborges@ufscar.br. https://orcid.org/0000-000 I-5941-4855. 
Objetivo: analizar evidencia cientifica nacional e internacional que aborde la percepción de los estudiantes de enfermería sobre el papel de las enfermeras en la Atención Primaria de Salud. Método: se trata de una revisión integrativa utilizando la base de datos de la Biblioteca de Salud Virtual y el análisis realizado por Estadisticas Textuales Clásicas y Clasificación Jerárquica Descendente de las conclusiones o consideraciones finales, utilizando el software IRAMUTEQ ${ }^{\circledR}$. Resultados: se seleccionaron 5 artículos de los 83 encontrados, y el análisis resultó en tres clases: Escasez de enfermeras para trabajar en la Atención Primaria de Salud; Educación universitaria vinculada a la Atención Primaria de Salud; y Percepción de los estudiantes sobre la profesión de enfermería. Conclusión: el proceso de educación y/o producción del conocimiento de enfermería sigue vinculando la profesión a las características subjetivas, señalando la necesidad de avanzar hacia la enfermería basada en la evidencia. También se observó la escasez de estudios primarios que abordan el tema de esta producción.

Descriptores: Estudiante de Enfermería. Atención Primaria de Salud. Estrategia de Salud Familiar. Enfermería. Revisión.

\section{Introdução}

O(a) enfermeiro(a), bem como os demais profissionais da saúde, possui um processo de trabalho a ser desenvolvido em sua atuação profissional. Este diz respeito ao modo como ele(a) executa, de maneira articulada, as ações assistenciais e gerenciais, por meio das relações de poder, dos saberes políticos, técnicos e filosóficos. Seu processo de trabalho responde a diversos fatores, como as necessidades fisiológicas dos seres humanos, demandas da coordenação do processo de trabalho de enfermagem e da organização do processo de trabalho em saúde inscrita em um determinado tempo histórico e social ${ }^{(1)}$. Tudo isso compõe a atuação desse(a) profissional nas diferentes áreas de atenção à saúde, tais como: cuidado domiciliar, setor hospitalar, Atenção Primária à Saúde (APS), dentre outras.

A APS consiste na porta de entrada desejável ao sistema de saúde e corresponde a um conjunto de ações individuais, coletivas e familiares, que envolvem a promoção, prevenção, diagnóstico, tratamento, reabilitação, redução de danos, cuidados paliativos e vigilâncias em saúde. Ela deve ser desenvolvida por uma equipe multiprofissional, sendo que um(a) dos(as) profissionais é o(a) enfermeiro(a) ${ }^{(2)}$.

O Brasil tem adotado a Estratégia Saúde da Família (ESF) como modelo de desenvolvimento da APS no país ${ }^{(2)}$ e, em 2018, a Organização Pan-Americana de Saúde/Organização Mundial de Saúde (OPAS/OMS) abordou a ampliação do papel do(a) enfermeiro(a) nessa instância de atenção, destacando suas funções ao apresentar o(a) enfermeiro(a) de prática avançada. Este(a) se diferencia no grau de autonomia na tomada de decisões, diagnóstico e tratamento das enfermidades acometidas pelos usuários dos serviços de saúde, atribuindo-lhe maior resolutividade na assistência à saúde da população (3).

Há, internacionalmente, um reforço constante às políticas e ações de saúde direcionadas aos cuidados desenvolvidos pelos(as) enfermeiros(as) de prática avançada na APS, pois eles proporcionam um alinhamento mais próximo às necessidades da população, à alta taxa de satisfação dos usuários e à otimização do custo-efetividade $^{(3)}$. Nesse sentido, também é função das universidades garantir a qualidade do cuidado prestado nessa instância de atenção à saúde, por meio de uma formação profissional articulada à realidade ${ }^{(4)}$.

Em se tratando dos estudantes de enfermagem, suas concepções sobre o trabalho do(a) enfermeiro(a) vai se modificando com o avançar do processo formativo e, sobretudo, com a sua inserção nos cenários de prática ${ }^{(5)}$. Tal fato, interfere diretamente na satisfação apresentada por eles(as) com o curso ${ }^{(6)}$, sendo um fator relevante na diminuição do estresse gerado pela própria formação acadêmica ${ }^{(7)}$.

Dessa forma, acreditamos que olhar para a percepção que os estudantes de enfermagem possuem sobre o trabalho do(a) enfermeiro(a) 
na ESF poderá contribuir diretamente com a ampliação do processo de formação profissional desses estudantes. Além disso, fornece pistas para o fortalecimento do processo formativo universitário na direção da atuação deste profissional nessa instância de atenção à saúde.

Diante dessa problemática, justifica-se o desenvolvimento da presente revisão integrativa, cujo objetivo foi analisar as evidências científicas nacionais e internacionais que abordam a percepção de estudantes de enfermagem sobre a atuação do(a) enfermeiro(a) na Atenção Primária à Saúde.

\section{Método}

Trata-se de revisão integrativa de literatura utilizando a estratégia Population, Intervention, Comparation, Outcome (PICO) para elaboração da questão norteadora da busca ${ }^{(8)}$. Assim, definimos para este estudo: P - estudantes de enfermagem, I - percepção dos estudantes de enfermagem sobre a atuação do enfermeiro(a) na APS, C - não foi aplicado neste estudo por não presumir um processo comparativo, $\mathrm{O}$ - atuação do enfermeiro na APS. Dessa forma, chegou-se à seguinte questão de pesquisa: Quais as evidências científicas que abordam a percepção de estudantes de enfermagem sobre a atuação do enfermeiro na APS?

Foram adotados como critérios de inclusão artigos científicos completos disponíveis on-line e publicados nos idiomas português, inglês e espanhol. Foram excluídos deste estudo dissertações, teses, literatura cinzenta e projetos de pesquisa ainda em desenvolvimento.

Como estratégia de busca, foi utilizada a Biblioteca Virtual em Saúde (BVS) com todas as suas bases de dados. Foi empregado o descritor controlado "estudante de enfermagem" em associação ao descritor "atenção primária à saúde" nos idiomas português, inglês e espanhol, combinados por meio do operador booleano $A N D$. A busca foi realizada no mês de fevereiro de 2020.

Os estudos encontrados foram criteriosamente avaliados, procedendo-se a seleção daqueles que se enquadravam nos critérios de inclusão e que possuíam relação com a questão estabelecida nesta revisão integrativa, sendo excluídos os demais.

Levando em consideração que as revisões integrativas demandam métodos normatizados e sistemáticos para garantir o rigor requerido e a legitimidade das evidências científicas obtidas ${ }^{(9)}$, foi elaborado um instrumento para auxiliar na identificação e análise dos dados dos estudos incluídos, composto pelos seguintes itens: Ano de publicação; Revista de publicação; Local de desenvolvimento da pesquisa; Título do artigo; Objetivo principal do estudo; Abordagem metodológica utilizada; e Conclusões ou Considerações Finais apontadas nas pesquisas.

Seguindo o processo Prisma para a seleção das produções, foram encontrados 83 arquivos, sendo que 4 estudos foram excluídos por se encontrarem em duplicidade. Portanto, 79 arquivos foram selecionados para aplicação dos critérios de inclusão e exclusão por meio da leitura dos títulos e/ou resumos, resultando em 6 artigos, que foram selecionados para leitura na íntegra por se enquadrarem na pergunta desta pesquisa. Após a leitura e utilização desse instrumento auxiliar, foi excluído um artigo por consistir em uma publicação referente a um projeto de pesquisa ainda em desenvolvimento, sem resultados e discussão dos dados. Assim, foram incluídos nesta revisão integrativa cinco artigos científicos (Fluxograma 1). 
Fluxograma 1 - Seleção dos artigos para a revisão integrativa

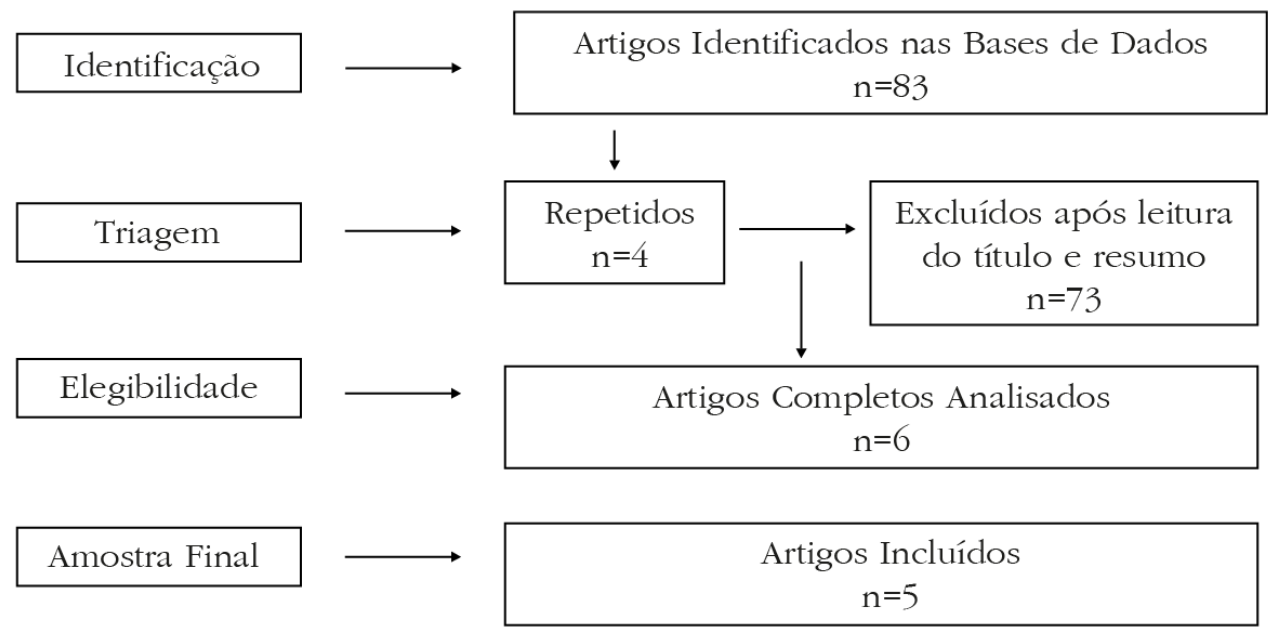

Fonte: Elaboração própria.

A análise dos dados ocorreu de duas formas: extração das informações contidas nas produções com o intuito de caracterizar a amostra de artigos incluídos na pesquisa; organização de um corpus textual por meio da junção dos textos apresentados nas conclusões ou considerações finais dos artigos, seguindo com seu processamento e análise por meio do software Interface de $R$ pour les Analyses Multidimensionnelles de Textes et de Questionnaires (IRAMUTEQ ${ }^{\circledR}$ ).

O IRAMUTEQ ${ }^{\circledR}$ realiza uma análise estatística mediante o agrupamento de vocábulos com similaridade semântica presentes no corpus textual. Esse corpus é dividido em seguimentos de textos (ST) pelo software, que consistem em pequenos fragmentos textuais que preservam a relação semântica entre si $^{(10)}$.

Assim, as conclusões ou considerações finais dos artigos selecionados foram traduzidas para o português (com exceção dos artigos já escritos nessa língua) e organizadas de forma a compor o corpus textual, preparado e revisado com o intuito de eliminar equívocos de digitação e padronização de siglas e expressões (preservando os mesmos significados). Foram incluídos adjetivos, advérbios, substantivos, verbos e formas não reconhecidas como categorias de palavras para o processo de análise pelo software e a análise foi realizada por Estatística Textual Clássica e Classificação Hierárquica Descendente (CHD). As ocorrências de cada uma das classes na CHD foram ordenadas de acordo com o valor do teste de Qui-Quadrado ( $\left.\mathrm{x}^{2}>3,80\right)$, de forma decrescente, e todas apresentaram valores estatisticamente significantes $(\mathrm{p}<0,05)$.

\section{Resultados}

As produções que compuseram este estudo, puderam ser caracterizadas segundo o ano de publicação: duas em 2016, duas em 2017 e uma em 2019; o país onde a pesquisa foi desenvolvida: duas na Austrália, uma no México e duas no Brasil (uma na Região Nordeste e outra na Região Sudeste); o idioma: três em inglês, uma em espanhol e uma em português; as abordagens metodológicas utilizadas: três pesquisas quantitativas transversais e duas pesquisas qualitativas, sendo uma destas utilizando o referencial teórico da fenomenologia. O consolidado dos artigos selecionados está apresentado em forma de quadro síntese com o ano de publicação, nome da revista, título do artigo, país onde a pesquisa foi desenvolvida, objetivo principal e abordagem metodológica utilizada (Quadro1). 
Quadro 1 - Caracterização dos estudos que abordam a percepção de estudantes de enfermagem sobre a atuação do enfermeiro na Atenção Primária à Saúde

\begin{tabular}{|c|c|c|c|c|c|}
\hline Ano & Revista & Título & $\begin{array}{l}\text { País da } \\
\text { Pesquisa }\end{array}$ & Objetivos & Metodologia \\
\hline 2016 & $\begin{array}{l}\text { Revista de } \\
\text { Enfermagem } \\
\text { do Centro- } \\
\text {-Oeste } \\
\text { Mineiro }\end{array}$ & $\begin{array}{l}\text { Percepção dos } \\
\text { estudantes de } \\
\text { graduação em } \\
\text { enfermagem sobre } \\
\text { o trabalho do } \\
\text { enfermeiro }\end{array}$ & Brasil & $\begin{array}{l}\text { Identificar a } \\
\text { percepção que os } \\
\text { estudantes de } \\
\text { enfermagem } \\
\text { possuem acerca } \\
\text { do trabalho do } \\
\text { enfermeiro }\end{array}$ & $\begin{array}{l}\text { Pesquisa } \\
\text { qualitativa, } \\
\text { utilizando de } \\
\text { entrevista semi- } \\
\text { estruturada com } \\
33 \text { estudantes de } \\
\text { enfermagem de } \\
\text { uma universidade } \\
\text { do nordeste } \\
\text { brasileiro }\end{array}$ \\
\hline 2016 & $\begin{array}{l}\text { Revista de } \\
\text { Pesquisa: } \\
\text { Cuidado é } \\
\text { Fundamental } \\
\text { Online }\end{array}$ & $\begin{array}{l}\text { The theory into } \\
\text { practice: teaching- } \\
\text { service dialogue } \\
\text { in the context of } \\
\text { primary healthcare } \\
\text { in the training of } \\
\text { nurses }\end{array}$ & Brasil & $\begin{array}{l}\text { Compreender as } \\
\text { percepções dos } \\
\text { alunos do curso } \\
\text { de graduação em } \\
\text { enfermagem sobre } \\
\text { sua inserção na } \\
\text { prática dos serviços } \\
\text { de atenção primária à } \\
\text { saúde. }\end{array}$ & $\begin{array}{l}\text { Pesquisa } \\
\text { qualitativa de } \\
\text { abordagem } \\
\text { fenomenológica } \\
\text { com } 9 \text { estudantes } \\
\text { do último ano de } \\
\text { uma universidade } \\
\text { estadual do } \\
\text { sudeste brasileiro }\end{array}$ \\
\hline 2017 & $\begin{array}{l}\text { Nurse } \\
\text { Education } \\
\text { Today }\end{array}$ & $\begin{array}{l}\text { Autralian student } \\
\text { nurse's knowledge } \\
\text { of and attitudes } \\
\text { towards primary } \\
\text { health care: a } \\
\text { cross-sectional study }\end{array}$ & Austrália & $\begin{array}{l}\text { Investigar o } \\
\text { conhecimento e } \\
\text { atitudes de estudantes } \\
\text { de enfermagem } \\
\text { australianos em } \\
\text { relação à abordagem } \\
\text { da atenção primária à } \\
\text { saúde }\end{array}$ & $\begin{array}{l}\text { Pesquisa } \\
\text { quantitativa, } \\
\text { descritiva, } \\
\text { transversal, com } \\
286 \text { estudantes } \\
\text { de enfermagem } \\
\text { de duas } \\
\text { universidades } \\
\text { australianas } \\
\end{array}$ \\
\hline 2017 & $\begin{array}{l}\text { Nurse } \\
\text { Education } \\
\text { Today }\end{array}$ & $\begin{array}{l}\text { Factors associated } \\
\text { with final year } \\
\text { nursing students' } \\
\text { desire to work in } \\
\text { the primary health } \\
\text { care settings: } \\
\text { findings from a } \\
\text { national cross- } \\
\text { sectional survey }\end{array}$ & Austrália & $\begin{array}{l}\text { Identificar os fatores } \\
\text { associados ao desejo } \\
\text { dos estudantes } \\
\text { de enfermagem } \\
\text { do último ano } \\
\text { em trabalhar na } \\
\text { atenção primária } \\
\text { à saúde, incluindo } \\
\text { fatores demográficos, } \\
\text { expectativas de } \\
\text { futuras condições de } \\
\text { emprego e conteúdo } \\
\text { do trabalho }\end{array}$ & $\begin{array}{l}\text { Pesquisa } \\
\text { quantitativa, } \\
\text { descritiva, } \\
\text { transversal, com } \\
530 \text { estudantes } \\
\text { de enfermagem } \\
\text { do último ano de } \\
14 \text { universidades } \\
\text { australianas }\end{array}$ \\
\hline 2019 & $\begin{array}{l}\text { Revista } \\
\text { Ciencia y } \\
\text { Cuidado }\end{array}$ & $\begin{array}{l}\text { Expectativas } \\
\text { académicas y } \\
\text { laborales en } \\
\text { estudiantes de } \\
\text { enfermería }\end{array}$ & México & $\begin{array}{l}\text { Conhecer as } \\
\text { expectativas } \\
\text { acadêmicas e laborais } \\
\text { de estudantes } \\
\text { que cursam o } \\
\text { último semestre } \\
\text { de licenciatura em } \\
\text { enfermagem, em uma } \\
\text { Universidade Pública } \\
\text { da Cidade do México }\end{array}$ & $\begin{array}{l}\text { Pesquisa } \\
\text { quantitativa, } \\
\text { descritiva, } \\
\text { exploratório e } \\
\text { transversal, com } \\
105 \text { estudantes de } \\
\text { enfermagem de } \\
\text { uma universidade } \\
\text { da Cidade do } \\
\text { México }\end{array}$ \\
\hline
\end{tabular}

Fonte: Elaboração própria. 
Com relação ao processo de análise das Conclusões ou Considerações Finais dos artigos, o corpus geral foi constituído por 5 textos, separados em 27 ST, com aproveitamento de 20 segmentos de texto (ST) (74,07\%). Emergiram 1.001 ocorrências (palavras, formas ou vocábulos), sendo 365 palavras distintas e 233 com uma única ocorrência. O conteúdo analisado foi categorizado em três classes: Classe 1, com 5 ST (25\%); Classe 2, com 6 ST (30\%) e Classe 3, com 9 ST (45\%).
Essas três classes foram divididas em duas ramificações (A e B) do corpus total em análise. O subcorpus A é composto pelas Classes 1 (Escassez de enfermeiros(as) para atuarem na APS) e 2 (Formação universitária vinculada à APS) e o subcorpus B, composto pela Classe 3 (Percepção dos estudantes sobre a profissão de enfermagem). Em seguida, apresentamos a explicitação dessa divisão, subdivisão e interrelação entre as classes (Fluxograma 2).

Fluxograma 2 - Dendograma das tendências dos artigos científicos - Classificação Hierárquica Descendente elaborado pelo software IRAMUTEQ

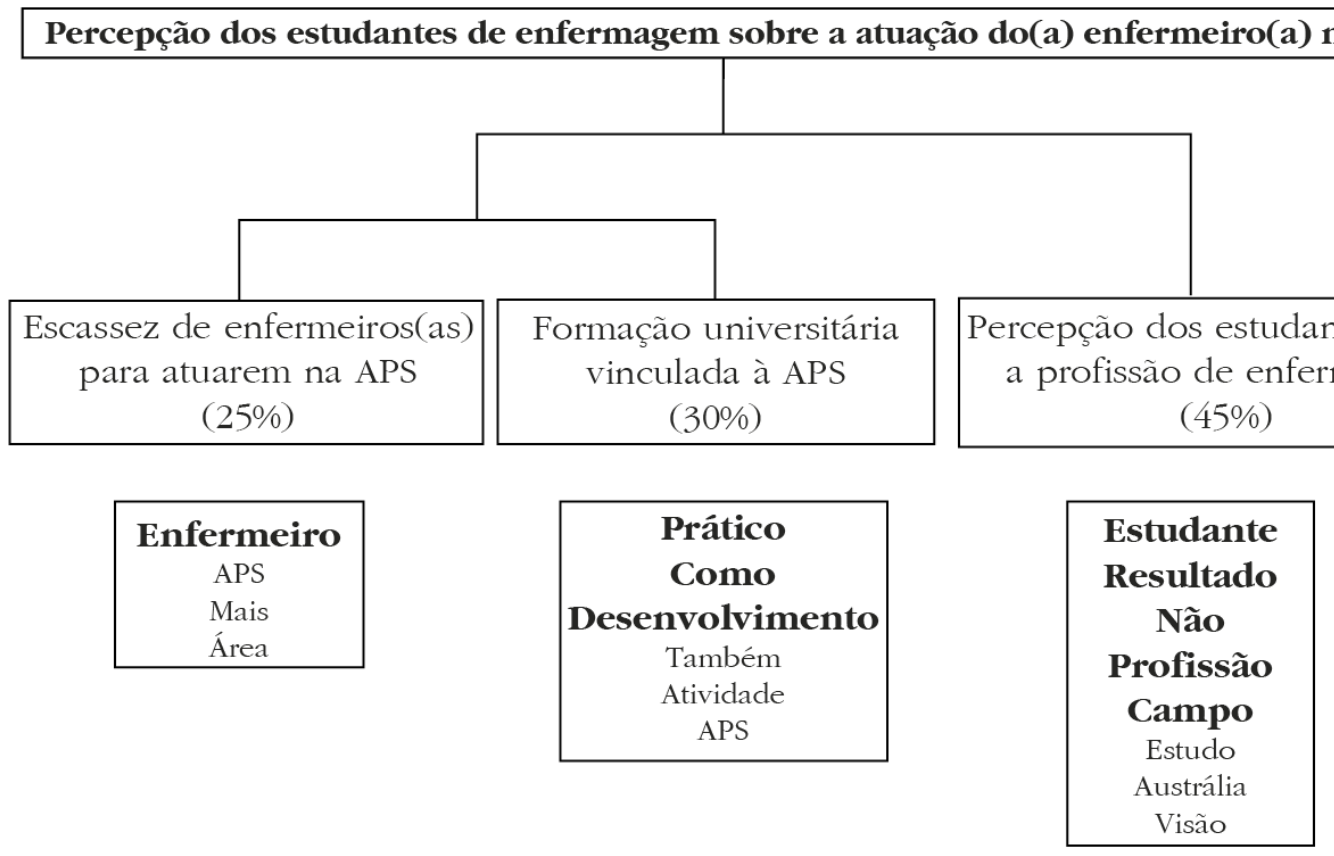

Fonte: Elaboração própria.

O subcorpus A se refere à "Enfermagem na APS", sendo que a Classe 1 foi constituída pela palavra e radical "enfermeiro" $\left(x^{2}=10\right)$ e corresponde à escassez de enfermeiros(as) atuando na APS, mesmo diante de um contexto sanitário mundial que requer essa especificidade de atenção à saúde da população. Alguns fragmentos de textos exemplificam a categorização dessa classe: "A APS é uma área que requer atenção imediata no que diz respeito ao desenvolvimento de enfermeiros qualificados [...] dada a escassez de enfermeiros que trabalham na APS e as necessidades atuais e futuras de assistência à saúde da população"(11:13). Outro fragmento de texto: "A necessidade de aprofundar discussões é enfatizada especialmente nas universidades e no SUS bem como o desempenho de estudos mais específicos sobre a formação de enfermeiros na APS, que são escassos" "(12:5008).

A Classe 2 foi constituída pelas palavras e radicais "prático" ( $\left.x^{2}=7,98\right)$, "como" $\left(x^{2}=5,71\right)$ e "desenvolvimento" ( $\left.\mathrm{x}^{2}=4,82\right)$ e corresponde ao processo da formação universitária de estudantes de enfermagem estar vinculada à APS, pois esse processo incentiva a existência de enfermeiros(as) aptos a atuarem nessa instância de 
atenção à saúde, bem como desenvolve outros domínios profissionais nos estudantes, tais como o vínculo e a compreensão do indivíduo em sua totalidade. Seguem alguns fragmentos de texto que elucidam essa classe: "O reconhecimento dos determinantes do processo saúde-doença, o vínculo a visão holística do paciente [...] em direção a ações inovadoras que vão além da técnica e que podem contribuir com subsídios para reverter o modelo de assistência médico-hospitalar ainda em uso"(12:5008). "O sistema de saúde pode ou não ser baseado no modelo biomédico, podendo afetar as atitudes das pessoas em relação à APS"(13:131).

O subcorpus B é composto pela Classe 3 constituída pelas palavras e radicais "estudante" ( $\left.x^{2}=9,9\right)$, "resultado" ( $\left.x^{2}=6,11\right)$, "não" $\left(x^{2}=4,31\right)$, "profissão" ( $\left.x^{2}=4,31\right)$ e "campo" $\left(x^{2}=4,31\right)$ e dizem respeito às percepções apresentadas pelos estudantes de enfermagem sobre a profissão, algo voltado para o cuidado, sobretudo no ambiente hospitalar, como demonstram alguns fragmentos de textos de alguns artigos: "A análise das descrições revelou que os estudantes percebem a profissão como algo que requer do profissional carinho e amor ao próximo"(14:2427). "As expectativas acadêmicas e profissionais dos estudantes que participaram desta pesquisa estão focadas no campo clínico, principalmente, em hospitais"(15:67). "Na Austrália, sabemos que a maioria dos estudantes prefere trabalhar em ambientes hospitalares" ${ }^{\text {(11:13). }}$

\section{Discussão}

É perceptível o escasso número de artigos científicos frutos desta revisão integrativa. Tal fato vai ao encontro daquilo que a totalidade dos artigos apontaram: a necessidade do desenvolvimento de pesquisas primárias sobre o tema, além de um olhar direcionado ao processo formativo de estudantes de enfermagem no que tange à APS.

O número de estudos quantitativos e qualitativos encontrados reflete, de certa forma, a necessidade de ambas abordagens na construção do conhecimento científico, as particularidades metodológicas apresentadas em cada uma e a complementariedade que elas possuem na explicitação de resultados e construção de hipóteses a serem desvendadas em estudos futuros. Tal constatação aponta para a potencialidade de estudos que contemplem métodos quantitativos e qualitativos, pois estes possibilitam o desenvolvimento de pesquisas que produzem dados que favorecem uma interpretação do fenômeno investigado com maior completude e abrangência $^{(16)}$.

Em se tratando das contribuições das pesquisas, os cinco artigos foram claros em seus aportes para o conhecimento da área, sendo que três deles (um estudo quantitativo(13) e os dois estudos qualitativos ${ }^{(12,14)}$ ) apresentaram especificamente a necessidade de ampliar para além da realidade local, por considerarem que os resultados apresentados não necessariamente poderiam ser generalizáveis a outros contextos. Chama a atenção que os dois estudos de abordagem qualitativa encontrem-se inseridos nessa conjuntura. Apesar de não consistirem em uma amostra significativa perante a gama de estudos qualitativos, eles trazem à tona a problemática das generalizações que essa abordagem possibilita (ou não) ao campo da ciência. Contudo, há concordância que apesar dessas produções enfocarem o universo microssocial e o aprofundamento dos fenômenos humanos, possibilitam compreender processos semelhantes que acontecem com os seres humanos frente aos mesmos desafios, possibilitando, portanto, generalizações, mas não na mesma perspectiva da significância estatística $^{(17)}$.

Um dos estudos apontou para a necessidade de um olhar crítico para a estrutura curricular dos cursos de graduação em enfermagem, associando esse processo a um possível aperfeiçoamento da formação direcionada à APS $^{(15)}$. Outro, concluiu que a maioria dos estudantes de enfermagem preferem trabalhar em ambientes hospitalares, carecendo de projetos por parte de diversas esferas para o desenvolvimento de iniciativas que aumentem a atratividade da APS como área de atuação do(a) enfermeiro(a) ${ }^{(11)}$. Tal constatação vai ao encontro de outros 
estudos científicos que vêm apontando para a grande concentração de profissionais da saúde em áreas urbanas e em serviços de atenção terciária (hospitalar) ${ }^{(18-21)}$. Somado a isso, existe uma grande disparidade no índice "profissionais médicos(as) e enfermeiros(as) por habitante" quando comparamos países desenvolvidos com países em desenvolvimento. Nos Estados Unidos e Reino Unido, por exemplo, essa proporção chega a 12,7 e 12,3, respectivamente, enquanto que na África Subsaariana é menor que 1,1 médico(a) e enfermeiro(a) para cada mil habitantes ${ }^{(19)}$. Com relação aos profissionais de enfermagem, há uma distribuição desigual tanto dentro quanto entre os diversos países do mundo. Na metade dos países, o índice de enfermeiros por dez mil habitantes é menor ou igual a 10,4. Da mesma forma, existe uma grande diferença quando comparamos esse mesmo índice entre o Canadá $(106,2)$, os Estados Unidos $(111,4)$ e Cuba $(81,3)$ com outros países do mundo ${ }^{(18-19)}$.

Dessa forma, é perceptível a escassez de enfermeiros(as) na atenção à saúde da população mundial, sendo que esse déficit é ainda maior quando direcionamos o olhar para a APS. Essa constatação também é perceptível na Classe 1 deste estudo, o que aponta para a necessidade de um triplo investimento para fomentar a perspectiva da abertura de novas vagas e oportunidades de emprego nessa instância de atenção à saúde; o aperfeiçoamento da formação dos(as) enfermeiros(as) para uma abordagem qualificada de atenção à saúde no contexto da APS; e a melhoria da sua capacidade técnica na resolução dos casos dentro da APS.

Em 2017, a Organização Mundial de Saúde (OMS) realizou uma reunião que apontou para a necessidade de avanços na qualificação técnica dos profissionais de enfermagem ${ }^{(22)}$, além do próprio movimento Nursing Now, que recomenda a colaboração entre diversos organismos internacionais para elevar a representação da enfermagem, elaborando um perfil de habilidades e competências para a sua atuação na APS, que inclui: prática clínica; liderança; experiência na atenção ao usuário, família e comunidade; investimento na promoção da saúde; desenvolvimento de ações interprofissionais e decisões pautadas na ética ${ }^{(4,23-24)}$.

Em se tratando do apresentado na Classe 2, estudos primários ${ }^{(25-26)}$ e revisão sistemática de literatura científica ${ }^{(27)}$ apontam para a potencialidade que a atividade prática no contexto dos serviços de saúde desempenha sobre a formação profissional no que tange à promoção de habilidades, atitudes e conhecimentos específicos para a atuação nessa instância de atenção à saúde.

No XVI Seminário Nacional de Diretrizes para a Educação em Enfermagem e no XIII Simpósio Nacional de Diagnóstico de Enfermagem, ocorrido em Florianópolis, em 2018, e promovido pela Associação Brasileira de Enfermagem, foi elaborada uma carta com recomendações que reforçam a relevância da integração ensino-serviço-comunidade desde o início do curso, inserindo os estudantes nos cenários de prática, articulando-os ao cotidiano vivenciado pelos profissionais de saúde. Antes disso, a edição especial de 2010 do periódico The Lancet ressaltou a relevância do sistema de saúde estar integrado ao sistema educacional, reforçando sua importância na composição do processo formativo (4) Tais apontamentos corroboram a Classe $2 \mathrm{da}$ presente produção, que apresenta a necessidade de uma formação universitária atrelada ao contexto real da APS.

Somado a isso, para que haja uma formação profissional de enfermeiros(as) condizente com o contexto atual que visa garantir maior autonomia profissional, faz-se necessário um processo formativo que leve em consideração as expectativas do(a) enfermeiro(a) com relação ao seu papel profissional e social, bem como a identificação das necessidades de saúde da população, fortalecendo sua inclusão em discussões curriculares e em pesquisas que levam em consideração a realidade social e política do país ${ }^{(28)}$.

Em se tratando das expectativas com relação à profissão de enfermagem, o presente estudo apresentou na Classe 3 o ambiente hospitalar e o carinho e amor ao próximo como algumas das características da profissão apontadas por estudantes de enfermagem. Estudo desenvolvido na Argentina, com 194 estudantes de enfermagem, 
apontou para o fato de que, durante a formação profissional, novos elementos são integrados às representações sociais dos estudantes, transformando-as e tornando-as mais complexas. Dessa forma, mudanças representativas acontecem na formação universitária quando se compara estudantes que entram com os que finalizam suas formações na Universidade ${ }^{(29)}$.

Outro estudo, desenvolvido na Holanda, com 240 estudantes de enfermagem, concluiu que a área hospitalar é a opção de preferência dos recém-formados (cerca de 70\% dos participantes). Esta constatação coloca em evidência a necessidade de uma atuação docente mais incisiva, estimulando uma formação universitária mais comunitária e vinculada à $\mathrm{APS}^{(30)}$. Tal fato retoma $\mathrm{O}$ apontamento feito sobre a necessidade de haver maior investimento na formação de enfermeiros(as), que garanta uma abordagem coerente e qualificada de atenção à saúde no contexto da APS, compreendendo que essa formação profissional acontece na Universidade, articulada ao contexto real dos serviços de saúde e atrelada diretamente à atuação dos profissionais da APS.

É relevante a articulação do exercício profissional às evidências científicas, com habilidades e atitudes ensinadas e aprendidas, e não como algo vinculado a características subjetivas e sentimentais do ser humano, como o carinho, o amor, a ternura e a afinidade, por exemplo. Esses sentimentos refletem em comportamentos de submissão e estão associados a uma carga histórica da profissão, valorizados e esperados naquela época. Contudo, isso parece "contribuir para que a profissão e o profissional não conquistem o devido respeito e continuem socialmente invisíveis" ${ }^{(31: 15)}$.

A limitação desta produção deveu-se ao corpus textual de análise ser relativamente pequeno, embora analisável e propositivo, pois contribuiu diretamente com a produção do conhecimento em enfermagem, evidenciando a escassa produção de estudos primários que abordem a percepção de estudantes de enfermagem sobre a atuação do(a) enfermeiro(a) na APS, e que envolvam essa perspectiva qualitativa na busca de compreender um pouco mais o perfil de profissionais de enfermagem que têm sido formados no contexto atual.

\section{Conclusão}

Os estudos primários existentes e analisados nesta revisão apontam para a pequena quantidade de enfermeiros(as) formados para atuarem na APS, a necessidade e os benefícios de uma formação universitária vinculada a essa instância de atenção à saúde, e que os estudantes de enfermagem percebem a profissão como um trabalho que utiliza do carinho, amor ao próximo e uma atuação mais voltada para o ambiente hospitalar.

Os resultados fazem transparecer que o processo de formação e/ou de produção do conhecimento em enfermagem ainda vincula a profissão a características subjetivas, precisando avançar na direção de uma enfermagem pautada em evidências científicas, com uma formação composta por habilidades e atitudes "ensináveis" e "aprendíveis".

Em suma, deve se acompanhar as tendências mundiais no que tange à formação do profissional enfermeiro(a), centrada em uma atenção à saúde autônoma, resolutiva e que reflita em benefícios concretos direcionados à sociedade.

\section{Colaborações:}

1 - Concepção, projeto, análise e interpretação dos dados: Pablo Ramon Rodrigues Freitas Ramos Carloni e Flávio Adriano Borges;

2 - Redação do artigo e revisão crítica relevante do conteúdo intelectual: Pablo Ramon Rodrigues Freitas Ramos Carloni, Amanda Caetano dos Santos e Flávio Adriano Borges;

3 - Aprovação final da versão a ser publicada: Pablo Ramon Rodrigues Freitas Ramos Carloni, Amanda Caetano dos Santos e Flávio Adriano Borges.

\section{Referências}

1. Mascarenhas NB, Santos TA, Florentino TC, Santos HS. Percepção de discentes, docentes e 
trabalhadoras sobre o processo de trabalho da enfermeira. Rev baiana enferm. 2019;33:e27930. DOI: $10.18471 /$ rbe.v33.27930

2. Brasil. Ministério da Saúde. Portaria no 2.436 , de 21 de setembro de 2017. Aprova a Política Nacional de Atenção Básica, estabelecendo a revisão de diretrizes para a organização da Atenção Básica, no âmbito do Sistema Único de Saúde (SUS) [Internet]. Brasília (DF); 2017 [cited 2020 May 11]. Available from: https://bvsms.saude.gov.br/bvs/ saudelegis/gm/2017/prt2436_22_09_2017.html

3. Cassiani SHB, Silva FAM. Ampliação do papel do enfermeiro na atenção primária à saúde: o caso do Brasil. Rev Latino-Am Enfermagem. 2019;27:e3245. DOI: $10.1590 / 1518-8345.0000 .3245$

4. Thumé E, Fehn AC, Acioli S, Fassa MEG. Formação e prática de enfermeiros para a atenção primária à saúde - avanços, desafios e estratégias para fortalecimento do Sistema Único de Saúde. Saúde Debate. 2018;42(esp 1):275-88. DOI: 10.1590/ 0103-11042018S118

5. Tonhom SFR, Moraes MAA, Pinheiro OL. Formação de enfermeiros na prática profissional: percepção de estudantes e professores. Rev Gaúcha Enferm. 2016;37(4):e63782. DOI: 10.1590/ 1983-1447.2016.04.63782

6. Ramos AC, Barlem JGT, Lunardi VL, Barlem ELD, Silveira RS, Bordignon SS. Satisfação com a experiência acadêmica entre estudantes de graduação em enfermagem. Texto Contexto Enferm. 2015;24(1):187-95. DOI: 10.1590/010407072015002870013

7. Bublitz S, Guido LA, Lopes LFD, Freitas EO. Associação entre estresse e características sociodemográficas e acadêmicas de estudantes de enfermagem. Texto Contexto Enferm. 2016;25(4):e2440015. DOI: 10.1590/010407072016002440015

8. Santos CMC, Pimenta CAM, Nobre MRC. A estratégia PICO para a construção da pergunta de pesquisa e busca de evidências. Rev Latino-Am Enfermagem [Internet]. 2007 [cited 2020 May 11]; 15:(3):508-11. Available from: http://www.scielo. br/pdf/rlae/v15n3/pt_v15n3a23.pdf

9. Soares CB, Hoga LAK, Peduzzi M, Sangaleti C, Yonekura T, Silva DRAD. Revisão integrativa: conceitos e métodos utilizados na enfermagem. Rev Latino-Am Enfermagem. 2014;48(2):335-45. DOI: $10.1590 /$ S0080-623420140000200020
10. Tomicic A, Bernardi F. Between Past and Present: The Sociopsychological Constructs of Colonialism, Coloniality and Postcolonialism. Integr Psychol Behav Sci. 2018;52(1):152-75. DOI: 10.1007/s12124017-9407-5

11. Bloomfield JG, Aggar C, Thomas THT, Gordon CJ. Factors associated with final year nursing students' desire to work in the primary health care setting: Findings from a national cross-sectional survey. Nurse Educ Today. 2018;61:9-14. DOI: 10.1016/ j.nedt.2017.10.001

12. Lima CA, Rocha JFD, Leite MTS, Santos AGP, Rodrigues BG, Lafetá AFM. A teoria em prática: interlocução ensino-serviço no contexto da atenção primária à saúde na formação do(a) enfermeiro(a). Rev Pesqui. 2016; 8(4):5002-9. DOI: 10.9789/2175-5361.2016.v8i4.5002-5009

13. Mackey S, Kwok C, Anderson J, Hatcher D, Laver S, Dickson C, et al. Australian student nurse's knowledge of and attitudes toward primary health care: a cross-sectional study. Nurse Educ Today. 2018;60:127-32. DOI: 10.1016/j.nedt.2017.10.003

14. Chagas SNF, Brito RS, Borges AMM. Percepção dos estudantes de graduação em enfermagem sobre o trabalho do enfermeiro. Rev Enferm Cent O Min. 2016;6(3):2421-9. DOI: 10.19175/recom.v6i3.1118

15. Escobar D, Covarrubias E. Expectativas académicas y laborales en estudiantes de enfermería. Rev cienc cuidad. 2019;16(2):59-71. DOI: $10.22463 / 17949831.1608$

16. Santos JLG, Erdmann AL, Meirelles BHS, Lanzoni GMM, Cunha VP, Ross R. Integração entre dados quantitativos e qualitativos em uma pesquisa de métodos mistos. Texto Contexto Enferm. 2017;26(3):e1590016. DOI: 10.1590/0104-07072017001590016.

17. Minayo MCS. Cientificidade, generalização e divulgação de estudos qualitativos. Ciênc saúde coletiva. 2017;22(1):16-7. DOI: 10.1590/141381232017221.30302016

18. Organização Pan-Americana da Saúde. Plataforma de Información en Salud par las Américas. Indicadores Básicos 2019: Tendencias de la Salud en las Américas [Internet]. Washington, D.C. (EUA); 2019 [cited 2020 May 11]. Available from: http:// www.paho.org/data/index.php/es/indicadores.html

19. Crisp N, Chen L. Global supply of health professionals. N Engl J Med. 2014;370(23):2247-8. DOI: 10.1056/NEJMra1111610 
20. Chen L, Evans T, Anand S, Boufford Jl, Brown H, Chowdhury M, et al. Human resources for health: overcoming the crisis. Lancet [Internet]. 2004; [cited 2020 May 11];364(9449):1984-90. Available from: http://www.globalsurgery.info/wp-content/ uploads/2014/01/Human-Resources-for-Health.pdf

21. Chen LC, Boufford JI. Fatal flows-doctors on the move. N Engl J Med. 2005;353(17):1850-2. DOI: 10.1056/NEJMe058188

22. Organização Mundial da Saúde. Report of the policy dialogue meeting on the nursing workforce [Internet]. Genebra (CH); 2017 [cited 2020 May 11]. Available from: http://www.who.int/hrh/news/ 2017/NursingApril2017-2.pdf?ua=1

23. Machado MH, Oliveira E, Lemos W, Lacerda WF, Aguiar Filho W, Wermelinger M, et al. Mercado de trabalho da enfermagem: aspectos gerais. Enferm Foco [Internet]. 2016 [cited 2020 May 11]; 7(spe):35-53. Available from: http://revista.cofen. gov.br/index.php/enfermagem/article/view/ $691 / 301$

24. Organização Pan-Americana da Saúde. Ampliação do papel dos enfermeiros na atenção primária à saúde [Internet]. Washington, D.C. (EUA); 2018 [cited 2020 May 11]. Available from: http://biblioteca. cofen.gov.br/wp-content/uploads/2018/05/ Ampliação-do-papel-dos-enfermeiros-na-atençãoprimária-à-saúde.pdf

25. Souza LB, Bonamigo AW. Integração ensino-serviço na formação de profissionais para sistemas públicos de saúde. Trab Educ Saúde. 2019;17(3):e0021747. DOI: 10.1590/1981-7746-sol00217

26. McComb SA, Kirkpatrick JM. Infusing systems and quality improvement throughout an undergraduate nursing curriculum. J Nurs Educ. 2017;56(12):752-7. DOI: $10.3928 / 01484834-20171120-10$

27. Markaki A, Moss J, Shorten A, Selleck C, Loan L, Mclain R, et al. Fortalecimento da saúde universal: desenvolvimento de um kit de ferramentas para melhoria da qualidade da educação em enfermagem e obstetrícia. Rev Latino-Am Enfermagem. 2019;27:e3188. DOI: 10.1590/15188345.3229 .3188

28. Toso BRGO, Padilha MI, Breda KL. O eufemismo das boas práticas ou a prática avançada de enfermagem. Esc Anna Nery. 2019;23(3):e20180385. DOI: 10.1590/2177-9465-EAN-2018-0385

29. Bastias F, Giménez I, Fabaro P, Ariza J, Caño-Nappa MJ. Social representations of nurses: differences between incoming and outgoing nursing students. Invest Educ Enferm. 2020;38(1):e05. DOI: 10.17533/ udea.iee.v38n1e05

30. Iersel MV, Vos R, Rijn MV, Latour CHM, Kirschner PA, Reimer WJMS. Influencing nursing students perceptions of community care with curriculumredesign, a quasi-experimental cohort study. BMC Med Educ. 2019;19:299. DOI: 10.1186/ s12909-019-1733-5

31. Lage CEB, Alves MS. (Des)valorização da enfermagem: implicações no cotidiano do enfermeiro. Enferm Foco. 2016 [cited 2020 May 11];7(3-4):12-6. Available from: http://revista. cofen.gov.br/index.php/enfermagem/article/ view/908/338

Recebido: 11 de maio de 2020

Aprovado: 4 de julho de 2020

Publicado: 25 de novembro de 2020

A Revista Baiana de Enfermagem utiliza a Licença Creative Commons - Atribuição-NãoComercial 4.0 Internacional. https://creativecommons.org/licenses/by-nc/4.0/

Este artigo é de acesso aberto distribuído sob os termos da Licença Creative Commons (CC BY-NC).

Esta licença permite que outros remixem, adaptem e criem a partir do seu trabalho para fins não comerciais. Embora os novos trabalhos tenham de lhe atribuir o devido crédito e não possam ser usados para fins comerciais, os usuários não têm de licenciar esses trabalhos derivados sob os mesmos termos. 